\title{
Bestimmung von Phenacetin und N-Acetyl-p-aminophenol über Stoffwechselprodukte im Harn ${ }^{1}$
}

\author{
Von H. Büch, H. Häuser, K. Pfleger und W. Rübiger \\ Aus dem Institut fïr Pharmakologie und Toxikologie (Direktor: Prof. Dr. W. Rummel) und dem Institut für Biochemie \\ (Direktor: Prof. Dr. H.-J. Bielig) der Universität des Saarlandes, Homburg/Saar
}

(Eingegangen am 7. Dezember 1965)

\begin{abstract}
Es wird eine Methode zum Nachweis und zur quantitativen Bestimmung von Phenacetin und N-Acetyl-p-aminophenol (NAPAP) nach Überführung ihrer Stoffwechselprodukte im Harn in p-Aminophenol angegeben. Die Bestimmbarkeitsgrenze liegt bei $20 \mathrm{mg} \%$, die Nachweisgrenze bei $1 \mathrm{mg} \%$ p-Aminophenol. Die Bildung eines gelben Phenoxazon-Derivates aus Phenacetin erlaubt zu entscheiden, ob der Patient Phenacetin oder NAPAP eingenommen hat. Bereits therapeutische Einzeldosen von $200-600 \mathrm{mg}$ dieser Analgetica sind bis zu 12 Stunden nach Einnahme bestimmbar, bis zu 48 Stunden nachweisbar.
\end{abstract}

A method is described for the detection and quantitative determination of phenacetin and N-acetyl-p-aminophenol (NAPAP); their urinary metabolites are converted into p-aminophenol. The limit of the quantitative determination is $20 \mathrm{mg} \%$ of $\mathrm{p}$-aminophenol; $1 \mathrm{mg} \%$ can be detected. From the formation of a yellow phenoxazone derivative from phenacetin, it is possible to decide whether the patient has taken phenacetin or NAPAP. Single therapeutic doses of $200-600 \mathrm{mg}$ of these analgetics can be determined up to 12 hours after dosage and detected up to 48 hours.

Die Schwierigkeiten beim Nachweis von Phenacetin und N-Acetyl-p-aminophenol („NAPAP“) beruhen darauf, $\mathrm{da} ß$ sie nur zum geringeren Teil unverändert im Harn auftreten; Umwandlungsprodukte machen den größten Teil der ausgeschiedenen Menge aus. Untersuchungen von Brodie und AXelrod (1) sowie von SMTth und Williams (2) zeigen, daß nach der Körperpassage von Phenacetin als Hauptausscheidungsprodukt (bis zu $80 \%$ der verabreichten Dosis) NAPAP — überwiegend konjugiert - im Harn erscheint. Auch nach Verabreichung von NAPAP selbst, das aufgrund seiner wesentlich geringeren Toxizität $(3,4)$ zunehmend häufiger in Kombinationspräparaten verwendet wird, treten die gleichen Konjugate auf. - Bisher beschriebene Nachweismethoden für diese Analgetica $(1,2,5)$ beruhen entweder auf dem Auswägen der isolierten Ausscheidungsprodukte oder auf Farbreaktionen der durch Hydrolyse erhaltenen freien Aminoverbindungen nach deren Trennung durch Verteilungsverfahren. Diese Methoden erfordern jedoch relativ viel Material, sind langwierig und zum Teil unspezifisch.

Hier wird eine einfache, schnelle und genaue Bestimmungsmethode angegeben, bei der man mit etwa $1 \mathrm{~m} l$ des zu untersuchenden Harns auskommt. Das Prinzip der Methode ist folgendes: nach Einnahme von Phenacetin oder NAPAP ist im Harn nach Hydrolyse mit Salzsäure das gleiche Spaltprodukt, nämlich $p$-Aminophenol, enthalten. Es entsteht aus freiem NAPAP durch Abspaltung der Acetylgruppe und aus den Konjugaten durch zusätzliche Abspaltung des Sulfat- bzw. GlucuronidRestes. Nach der dünnschichtchromatographischen Auftrennung des Urinhydrolysates ist p-Aminophenol aufgrund seiner UV-Absorption und seiner Braunfärbung an der Luft nachweisbar. Es kann nach Elution durch spektrophotometrische Messung der Extinktion bei $240 \mathrm{~m} \mu$ quantitativ bestimmt werden.

1) Ein Teil dieser Ergebnisse wurde auf der 6. Frühjahrstagung der Deutschen Pharmakologischen Gesellschaft, Mainz 1965, vorgetragen.

\section{Methode}

Hydrolyse und Dünnschichtchromatographie

$0,8 \mathrm{ml}$ Urin werden nach Zugabe von $0,2 \mathrm{ml}$ konz. Salzsäure in einem Reagenzglas eingeschmolzen. Die Hydrolyse wird bei $110^{\circ}$ im Trockenschrank während $90 \mathrm{Min}$. durchgeführt. Danach werden ansteigende Mengen $(0,02,0,05$ und $0,10 \mathrm{~m} /)$ des Hydrolysates bandförmig (nicht mehr als $0,01 \mathrm{~m} l$ pro Auftragstelle) auf Dünnschichtplatten (Kieselgel $\mathrm{GF}_{254}$, Schichtdicke $0,25 \mathrm{~mm}$ ) aufgetragen. Die DC-Platten werden nach der Methode von STAHL (6) hergestellt und aufbewahrt. Die DC-Platten werden $30 \mathrm{Min}$. bei $105^{\circ}$ im Trockenschrank aktiviert.

Die Auftrennung des Hydrolysates erfolgt durch aufsteigende Chromatographie mit dem Fließmittel iso-Propanol/Chloroform/ 33-proz. Ammoniak (7:2:1). Eine Laufstrecke von $10 \mathrm{~cm}$ wird bei Kammersättigung in ungefähr 60 Min. erreicht.

\section{Qualitative und quantitative Erfassung}

Nach dem Trocknen der DC-Platten kann p-Aminophenol als dunkler Fleck auf dem im UV-Licht fluoreszierenden Untergrund anhand der mitgelaufenen Testsubstanz (z. B. gewonnen aus NAPAP durch Hydrolyse mit Salzsäure) leicht lokalisiert werden. Innerhalb von 1 bis 2 Stunden wird der p-Aminophenol-Fleck zunehmend braun und läßt sich dann auch im sichtbaren Licht erkennen. Zur weiteren Kennzeichnung kann die Blaufärbung mit dem Phenolreagenz von Folrn und Crocalteu herangezogen werden: nach Besprühen mit 5-proz. $\mathrm{Na}_{2} \mathrm{CO}_{3}$-Lösung und kurzem Trocknen wird eine 20-proz. wäßr. Lösung des Phenolreagenzes (Fa. Merck) aufgesprüht.

Zur quantitativen Bestimmung wird zunächst die Lage der pAminophenol-Flecke der aufgetragenen Proben im UV-Licht durch Umranden gekennzeichnet. Nach Ablösen des Kieselgels wird das p-Aminophenol jeweils mit $5 \mathrm{ml} 0,5 \mathrm{~N} \mathrm{NaOH}$ eluiert. Nach Klärung des Eluates durch Zentrifugieren wird die Extinktion in Quarzküvetten $(\mathrm{d}=1 \mathrm{~cm})$ mit einem Spektralphotometer (Zeiss PQM II) bei $240 \mathrm{~m} \mu$ gegen eine Blindprobe bestimmt. Zur Aufstellung einer Eichkurve (Ordinate $\log \mathrm{I}_{0} / \mathrm{I}$ bei $240 \mathrm{~m} \mu$ ) werden steigende Mengen einer frisch bereiteten Lösung von pAminophenol in $2 \mathrm{~N} \mathrm{HCl}(10-100 \mu \mathrm{g} /$ Fleck) auf DC-Platten aufgetragen, chromatographiert und bestimmt.

\section{Ergebnisse und Diskussion}

Nach Einnabme von $N A P A P$ findet man nach dünnschichtchromatographischer Auftrennung des Urinhydrolysates nur $\mathrm{p}$-Aminophenol $\left(R_{F}=0,80\right)$. Die Nachweisbarkeitsgrenze beträgt im UV-Licht $2 \mu \mathrm{g} /$ Fleck, im sichtbaren Licht nach Braunfärbung $1 \mu \mathrm{g} /$ Fleck 
und nach Blaufärbung mit dem Phenolreagenz 0,1 $\mu \mathrm{g}$ p-Aminophenol/Fleck. $\mathrm{Da}$ gegenüber einer direkten Verdünnungsreihe von p-Aminophenol in $0,5 \mathrm{~N} \mathrm{NaOH}$ $\left(E_{1}^{1 \%} \mathrm{~cm}=900\right.$ bei $\left.240 \mathrm{~m} \mu\right)$ bei der Chromatographie ein Verlust von" etwa $10 \%$ an 'p-Aminophenol auftritt, empfiehlt es sich," ${ }^{*}$ stets eine Eichkurve aufzustellen. Die bei diesem Vorgehen erhaltene Streuung beträgt etwa $\pm 5 \%$. Der Verlust an p-Aminophenol unter den Hydrolysebedingungen $(<0,5 \%)$ kann vernachlässigt werden. Die untere Bestimmbarkeitsgrenze im Harn liegt bei $20 \mathrm{mg} \%$ p-Aminophenol. In $0,1 \mathrm{ml}$ Hydrolysat müssen also mindestens $15 \mu \mathrm{g}$ p-Aminophenol zur Messung nach dünnschichtchromatographischer Auftrennung verfügbar sein.

Nach Einnabme von Phenacetin findet man auf dem Dünnschichtchromatogramm des Urinhydrolysates außer pAminophenol noch mehrere Farbstoffe (grün $R_{F}=0,68$, violett $R_{F}=0,86$ und gelb $\left.R_{F}=0,96\right)$. Der gelbe Hauptfarbstoff, 3-Amino-7-äthoxy-phenoxazon-(2), den man auch nach kleinen Phenacetin-Dosen auf dem Chromatogramm erkennen kann, entsteht folgendermaßen: die primären Ausscheidungsprodukte des Phenacetins - p-Äthoxy-o-sulfonyloxy-anilin und pÄthoxy-o-hydroxy-acetanilid (7) - werden zu pÄthoxy-o-hydroxyanilin hydrolysiert. 2 Moleküle dieser Substanz kondensieren auf der DC-Platte im alkalischen Fließmittel zu dem angegebenen Phenoxazon-Derivat (8):

2

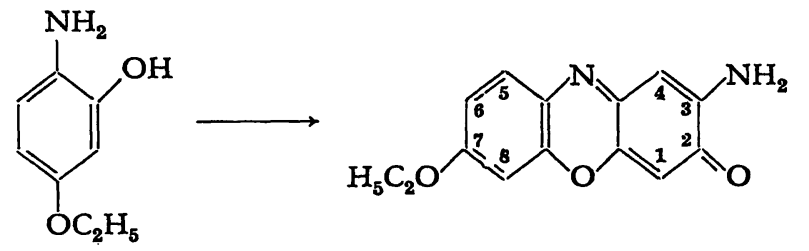

Ein Fehlen des Phenoxazon-Fleckes bedeutet also, daß das nachgewiesene p-Aminophenol nicbt aus Phenacetin entstanden sein kann.

p-Phenetidin $\left(R_{\mathrm{F}}=0,91\right)$, das nach Phenacetin-Einnahme im hydrolysierten Harn zusätzlich vorhanden ist (1) und unter unseren Bedingungen (durch die salzsaure Hydrolyse) aus unverändert ausgeschiedenem Phenacetin entsteht, kann man aufgrund seiner geringen Konzentration ( $<1 \%$, bez. auf die verabreichte Dosis) auf der DC-Platte unter den angegebenen Bedingungen nicht erkennen.

p-Aminophenol wird mit dem beschriebenen Fließmittel dünnschichtchromatographisch sicher von folgenden u. U. im Harn anwesenden Barbituraten abgetrennt: Phenobarbital $\left(R_{F}=0,48\right)$, Barbital $\left(R_{F}=\right.$ $0,60)$ und Methylphenobarbital $\left(R_{F}=0,64\right)$, die ein UV-Maximum zwischen 245 und $255 \mathrm{~m} \mu$ aufweisen. Thiopental $\left(R_{F}=0,77\right)$, das im Gegensatz zu den übrigen Barbituraten eine positive Folin-Ciocalteu-Reaktion (schwache Blaufärbung) gibt, wird von p-Aminophenol nicht getrennt. Die Unterscheidung gelingt über die UV-Absorptionskurve zwischen 360 und $230 \mathrm{~m} \mu$ : In $0,05 \mathrm{~N} \mathrm{NaOH}$ hat Thiopental ein einziges Maximum bei $305 \mathrm{~m} \mu, \mathrm{p}$-Aminophenol ein Hauptmaximum $\left(\lambda \max _{1}=\right.$ $240 \mathrm{~m} \mu$ ) und ein Nebenmaximum $\left(\lambda \max _{2}=315 \mathrm{~m} \mu\right)$ mit $E_{1}: E_{2}=3,7$.

\section{Anwendung}

Bei einer Reihe von Versuchspersonen wurde nach oraler Gabe von $1 \mathrm{~g}(5,6 \mathrm{mMol})$ Phenacetin bzw. $2 \mathrm{~g}$ $(13,2 \mathrm{mMol}) \mathrm{N}$-Acetyl-p-aminophenol (NAPAP) das in 8 bzw. 6 Stunden im Urin auftretende, nicht-konjugierte bzw. konjugierte NAPAP als Summe des durch Hydrolyse entstandenen p-Aminophenols gemessen (Tab. 1 u. 2).

Aus Tabelle 1 ist ersichtlich, $\mathrm{da} \beta$ in 8 Stunden bei Menschen mit einem mittleren Harnvolumen von $485 \mathrm{ml}$

Tab. 1: Ausscheidungsverhältnisse nach oraler Gabe von $1 \mathrm{~g}$ (5,6 mMol) Phenacetin (in 8 Stdn. ausgeschieden)

\begin{tabular}{cccc}
\hline $\begin{array}{c}\text { Versuchs- } \\
\text { person }\end{array}$ & $\begin{array}{c}\text { Harn } \\
\text { ml }\end{array}$ & $\begin{array}{c}\text { NAPAP } \\
\text { konjug. u. nicht-konjug. } \\
\text { (best. als p-Aminophenol) } \\
\text { mMol }\end{array}$ & $\begin{array}{c}\% \\
\text { der gegebenen Dosis }\end{array}$ \\
\hline I & 245 & 2,15 & 38 \\
II & 275 & 2,02 & 36 \\
& 1120 & 3,51 & 62 \\
\hline III & 300 & 1,00 & 39 \\
\hline
\end{tabular}

Tab. 2: Ausscheidungsverhältnisse nach oraler Gabe von $2 \mathrm{~g}$ (13,2 mMol) NAPAP (in 6 Stdn. ausgeschieden)

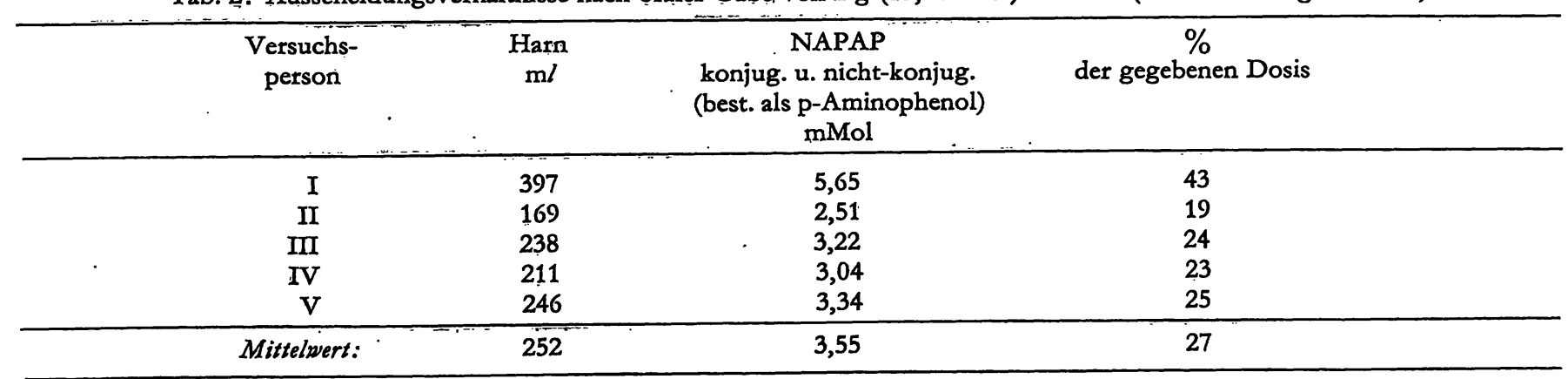


rund 39\% der verabreichten Phenacetin-Dosis als konjugiertes bzw. nicht-konjugiertes NAPAP mit der angegebenen Methode als p-Aminophenol erfaßt werden. Aus Tabelle 2 geht hervor, daß 6 Stunden nach Verabreichung von NAPAP sich in einem mittleren Harnvolumen von $252 \mathrm{~m} l$ rund $27 \%$ der verabreichten Dosis $(2 \mathrm{~g})$ mit der neuen Methode auffinden lassen. Da 20 $\mathrm{mg} \%$ p-Aminophenol im Harn quantitativ, $1 \mathrm{mg} \%$ noch qualitativ erfaßbar sind, ergibt sich, daß therapeutische Einzeldosen von $200-600 \mathrm{mg}$ Phenacetin bzw. NAPAP bis zu 12 Stunden nach Einnahme bestimmbar, bis zu 48 Stunden nach Einnahme nachweisbar sind. In allen Urinproben war nach Gabe von Phenacetin außerdem das gelbe Phenoxazon-Derivat nachweisbar.

Somit liegt eine Methode vor, die es gestattet, unter Zuhilfenahme der Dünnschichtchromatographie und der Spektrophotometrie sichere Angaben über die Einnahme von Phenacetin oder NAPAP zu machen. Bei einer Intoxikation mit diesen Analgetica läßt sich durch quantitative Bestimmung im 24-Stunden-Urin über mehrere Tage und Addition der gemessenen p-Aminophenol-Mengen die eingenommene NAPAP- oder Phenacetin-Dosis ziemlich genau ermitteln. Bei derartigen Bilanzuntersuchungen muß nach PhenacetinEinnahme berücksichtigt werden, daß die Ausscheidung an p-Äthoxy-o-sulfonyloxy-anilin in 8 Stunden rund $8 \%$ - bezogen auf die eingenommene Dosis - beträgt (7). Dagegen kann man die Ausscheidung von unverändertem Phenacetin und von p-Phenetidin - zusammen $<1 \%$, bezogen auf die eingenommene Dosis - die die p-Aminophenol-Bestimmung ebenfalls nicht erfaßt, vernachlässigen.

\title{
Literatur
}

1. Brodie, B. B. und J. Axelrod, J. Pharmacol. exp. Therapeut. 97, 58 (1949). - 2. SMith, J. N. und R. T. WiLLIAMs, Biochem. J. 44, 239 (1949). - 3. Pfleger, K., W. Rummel, E. Seifen und H. BleICHER, Arch. int. Pharmacodynam. Thérap. 134, 467 (1961). - 4. Clausen, E. und O. A. Larsen, Acta pahrmacol. toxicol. (København) 22, 135 (1965). - 5. Brodie, B. B. und J. Axelrod,
J. Pharmacol. exp. Therapeut. 94, $22 \cdot(1948)$. - 6. STAHL E., Dünnschichtchromatographie, S, 7-10, Springer-Verlag BerlinGöttingen-Heidelberg (1962). - 7. BücH, H., H. HäUSER, K. Pfleger und W. Rüpiger, Naunyn-Schmiedebergs Arch. exp. Pathol. Pharmakol. 253, 25 (1966). - 8. Büch, H., H. HÄUSER, K. PFLeger und W. RüDIGER (in Vorbereitung).

\section{Mikromethode zur quantitativen Chloridbestimmung}

\author{
Von F. C. StTzManN \\ Aus der Universitätskinderklinik Erlangen-Nürnberg (Direktor: Prof. Dr. A. Windorfer)
}

(Eingegangen am 23. Dezember 1965)

\begin{abstract}
Es wird über eine einfache, rasche und genaue Mikromethode zur Chloridbestimmung in Serum, Schweiß und Urin berichtet. Die Methode beruht auf der photometrischen Messung der durch Chlorionen freigesetzten Chloranilsäure aus Quecksilberchloranilat im sauren Medium. Die Zuverlässigkeit der Methode wurde überprüft. Die gefundenen Normalwerte liegen durch die Enteiweißung des Serums niedriger ( $81-103 \mathrm{mval} / \mathrm{l})$. Wegen der geringen Untersuchungsmenge kann diese Methode für die Kinderklinik empfohlen werden.

A simple, rapid and precise micromethod for the determination of chloride in serum, sweat and urine is described. The method is based on the photometric measurement of chloranilic acid, which is formed from mercury chloranilate in the presence of chloride ions in acid media. The reliability of the method is discussed. The method is recommended for the pediatric clinic, because the necessary amount of serum is small; the normal value, found by this method, is $81-103$ meq. $/ l$.
\end{abstract}

Die für quantitative Bestimmungen notwendigen Serummengen stehen in der Kinderklinik nicht immer zur Verfügung; bei Frühgeborenen und jungen Säuglingen bereitet ihre Gewinnung oft erhebliche Schwierigkeiten. Andererseits verlangen die Stoffwechselstörungen in dieser Altersklasse einen genauen Blick in die vorliegende Ionenverschiebung. Man sucht besonders in der Kinderheilkunde daher nach Mikro- und Ultramikromethoden. Auch zur Chloriduntersuchung im Schweiß, einer wichtigen Methode zur Diagnose der Mucoviscidose, wird man sich einer Mikromethode bedienen müssen, da die gewonnenen Schweißmengen oft unter $0,5 \mathrm{~m} l$ liegen (1). Bisher entwickelte Mikro- und Ultramikromethoden, die wir auch RichTERICH (2) verdanken, haben sich gut eingeführt und im Labor bewährt.
BARNEY (3) und BertolacinI (4) haben 1957 vorgeschlagen, die Chlorionen in der Reaktion mit dem Silbersalz der Chloranilsäure zu bestimmen. Die auf diese Weise freigesetzte Chloranilsäure wird dann photometrisch gemessen. BAZINSKI und Mitarbeiter (5) haben die Chloridbestimmung nach diesem Reaktionsschema in großen Untersuchungsreihen durchgeführt. Dabei wurde jedoch das Quecksilbersalz der Chloranilsäure genommen, da das entstehende Quecksilberchlorid nur wenig dissoziiert (modifizierte Methode nach LANG (6)). $\mathrm{Da}$ hierbei auch bei sehr wenig Untersuchungsmaterial zuverlässige Werte erhalten werden, haben wir diese Methode überprüft und vergleichende Untersuchungen mit der von uns bisher angewandten Methode vorgenommen, wobei die elektrometrische Titration mit 